\title{
An Adaptive Methodology to Overcome Localization Translation Challenges
}

\author{
Abbas Brashi ${ }^{1}$ \\ ${ }^{1}$ English Language Department, Umm Al-Qura University, Saudi Arabia \\ Correspondence: Abbas Brashi, English Language Department, Umm Al-Qura University, Saudi Arabia. E-mail: \\ asbrashi@uqu.edu.sa
}

Received: June 8, $2021 \quad$ Accepted: July 12, $2021 \quad$ Online Published: July 18, 2021

doi:10.5539/ijel.v11n4p105 URL: https://doi.org/10.5539/ijel.v11n4p105

\begin{abstract}
This study proposes an adaptive methodology to overcome localization translation challenges. The objective of the study is to generate a theoretical framework for identifying localization translation problems and ultimately propose a user-centred and agile-based methodology to minimize translation errors. The main research question that this paper attempts to answer is the question of "What would be the best theoretical framework for identifying current translation problems and addressing the convergence of translation and localization according to the new developments in informatics and communication technologies?" To answer this question, it was important to dismantle the notions of translation, translation theory, and localization. Based on the revised new definitions adapted to the new socio-technological context of the present digital era, the challenges can be identified and addressed through the formulation of a new methodology. The new methodology involves several steps, including the selection of recognized techniques like the "rich points" model to identify the localization translation challenges, a set of quality criteria to evaluate the projects, and adopting a user-centred approach and agile methodology for the project management of localization translation projects in order to assure the satisfaction of the stakeholders and a rapid adaptation to changes in the requirements. The proposed methodology must be validated in the future by applying it to concrete cases of localization translation projects and assessing its utility and performance. Thus, it would be useful in the future for improving localization translation projects.
\end{abstract}

Keywords: translation, localization, project management, agile, heuristic evaluation

\section{Introduction}

The field of translation is one of the most difficult fields to be defined. Richards (1953) even affirmed that translation is one of the most difficult aspects to be addressed in the entire evolution of everything. However, in the last century, there were many researchers who tried to reduce translation to a component of linguistics. For example, Shveitser (1987) made the claim that translation is an object of linguistic study. It seemed that translation theorists and linguists were following separate ways (Bell, 1991), with the translation theorists considering that the rigid framework of linguistics cannot contain the translation theories and with the linguistics considering that translation is a subdomain of linguistics or at least it can be entirely explained by it.

Although the translation theory is a very vast field of study and cannot be explained through linguistics, it is very important to understand the importance of linguistics to translation (Fawcett, 1997). Berman (1989) explained that translation can be talked about in many ways: translation can be considered an entity of study for disciplines like linguistics, comparative literature or poetics, as well as hermeneutics and, also, translation can be analyzed from an experiential point of view, connecting translation with philosophy and psychoanalysis.

Another notion related to the translation theory is the concept of localization. The relation between translation and localization is oftentimes addressed in academia, but, in case of some industries, the relation is not treated in a systematic way, but rather in an ad hoc manner (O'Hagan \& Mangiron, 2013). Generally speaking, there are not enough systematic studies to address the complex relationship between translation and localization taking into account at the same time the technological disruptions of the current digital era. Recently, the presence of international businesses on the internet has played a significant role in the growth of market shares and the expansion of their services. Those companies that have showcased their business activities on the internet have survived regardless of the latest economic crises and even made a profit (Lakó, 2014), for example the recent 
Covid-19 crisis that still exists. This highlights the significance of providing easy access of information for clients.

Due to the technological advances and of new ways of conducting marketing, the notion of localization becomes more blurry and it needs to be addressed. The two domains, translation and localization, tend to converge (O'Hagan \& Mangiron, 2013). Therefore, it is highly important to create new links between academia and industry. Many industries would benefit enormously from defining a more solid theoretical framework in which the relation between translation and localization is addressed.

Some of the greatest challenges occur when translating content into different languages in the context of a global market engaging customers all around the world. The aim of the current research is to attempt to create an adaptive methodology which can be useful for different industries in overcoming the social and technological challenges concerning translation and localization. The methodology would be able to make translation and localization much more efficient through the use of an iterative approach to the project management and an efficient process to mitigate the challenges related to the translation and localization in the current digital century.

\section{Literature Review}

\subsection{Definitions}

For addressing the current challenges concerning translation and localization, it is important to analyze how translation and localization are defined nowadays and what is the nature of the challenges.

As determined by Fawcett (1997), translation can be related to linguistics in two different ways: by applying theories of linguistics to the practice of translation (resulting in a linguistic theory of translation) or by applying the economic, literary and psychological theories of translation. Saussure (1972) came up with a very useful classification in understanding the relation between linguistics and translation, he distinguished between "langue" (the abstract language system, the object of linguistics) and "parole" (the actual uses of language, involving numerous random factors which cannot be reduced to an abstract system, therefore cannot be an object of linguistics).

Following the classification of Saussure (1972), Ladmiral (1979) also concluded that translation should be seen as a communication operation which can assure the same parole across different langues. Also, Koller (1979) declared that translation theory is "a science of parole." This view over translation theory as being a science of parole became very popular and Pergnier (1993) referred to it as a widely accepted truth, affirming that translation is actually a fact of parole, every translation being different, and a perfect translation not being possible.

Nord (2005) states that translation is an inter-subjective problem to solve. It is an interdisciplinary field, continuously evolving and bringing new theories to life (Odacioğlu, 2017). As the consumer software industry emerged, with that flourished the localization sector that was influenced by the production of personal computers during early 1980s (O'Hagan \& Mangiron, 2013). The concept of "localization" was initially introduced by software developers in the late 1980s, and its purpose was to adapt to the initial content, display, source code, or the foreign cultural-linguistic elements (Folaron, 2006). At the same time, the very first multi-language vendors appeared and were providing localization services. Soon, the publishers of software realized the level of complexity which localization projects have and they acknowledged the need for specialized knowledge in localizing software products (Esselink, 2000). Despite the fact that the establishment of localization industry has well flourished now, however, the specific vantage point of the definer still makes the definition of localization vary (O’Hagan \& Mangiron, 2013). Dunne (2006) observes that localization should not be seen as a global phenomenon as there is not yet a consensus over what it really is. Localization Industry Standards Association (LISA) originally came with the definition of localization as a vast concept, which can also be applied to translation, defining localization as a method of modifying the services or products in order to integrate the changes in different markets (Lommel, 2003). The term "localization" was generated from the word "locale", as explained by Esselink (2000), which means a small zone, or, in practical applications, an integration of several elements, like language, region, or character encoding.

Skopos theory marked a new trend of commercial web localization in translations studies (Vermeer, 2001). According to Lakó (2014), the main difference between a general text to be translated and web content translation is that the author of the former does not (generally) plan to be translated into several languages. The purpose of commercial web localization was to enhance the sales or traffic for source text and target text. The restrictions imposed by the web page design can result in the change of the original text. The web content 
translation author does not intentionally plan to translate the text, but localization translation on a commercial website works as a localization that is implemented globally on various locales. The internationalization of the text can be acquired by using more general language which is more understandable to the target audience.

In the functionalist theory by Nord (1997), the term web localization was broadly defined by the distinction between documentary and instrumental translation. Documentary translation is a type of translation method in which the source sender generates a documentation to create communication channel between himself and the target source, whereas instrumental translation serves as an instrument of communication with the source text target automatically, without giving a notice to the source text sender. Instrumental translation works efficiently by convincing the users and Nord (1997) called it a "function-preserving translation", which has three sub-branches: equifunctional, heterofunctional and homologous. Nord's theory of translation localization (Nord, 1997) is same as copywriting. The only difference between them is that the copywriter should be proficient in target language, whereas the translator must be capable of source language as well as the target language.

According to Mazur (2007), localization has an associated metalanguage, utilizing special words such as delta, MLVs (Multiple Language Vendors), simship, SLVs (Single Language Vendors), fuzzy matches and so on. Delta means the time gap between the release of the product or service in the original language and the release in another language or, in other words, between the release of the product or service in the lead market and the released in the localized market according to Lommel (2003), MLVs means multiple language vendors, simship means the simultaneous shipment of translation or simultaneous release (Sprung, 2000), SLVs means single language vendors, for example, the local translation agencies to which the work of translation is outsourced. Other useful terms are Translation Memory (TM) and Machine Translation (MT), which should not be confused. In case of TM, the translator uses previous translations to save time and most TM systems are called "fuzzy matches." MT, as the name suggests, is an automatic translation, done by computers, where the machine translation system analyzes the source text, breaks it down into parts and then reassembles them in the target language (Lommel, 2003).

\subsection{Translation and Localization Challenges}

There are many challenges regarding translation and localization, namely challenges given by the source text, by the lack of internationalization practices and nonadjustable translation tools (de la Cova, 2016), tight deadlines, conflict (Alonso, 2016), lack of clarity and trust between translators and managers and potential customers, which is due to the high level of digitalization of the communication channels and of translation production networks (Abdallah \& Koskinen, 2007). One of the most important challenges is the so-called translation memory segmentation process (de la Cova, 2016).

When these factors are not taken into account, the mistake can be deadly. One of the most famous examples of translation mistakes was the case of the Spanish fashion retailer Zara when they translated wrongly the Spanish "sandalias de esclava" into the German expression "dreifarbige Sklaven Sandalen." The term "esclava" refers to a particular style of bracelet in Spanish, but was translated literally into German as "slave" (Pym, 2014).

The challenges in localization translation can increase as well due to other types of considerations. For example, there are some interesting situations when a strategy of non-translation is adopted. The world has become more homogeneous and, for example, a French name of a perfume should not be translated, otherwise it will lose its aura of sophistication and luxury. In other situations, some words which are not relevant are even omitted. Cronin (2013) stated that translation is what makes globalization a reality.

Another interesting trend is that the local brands and products aim to be both local and international at the same time (Rodrigues, 2016). Thus, there is a tension to preserve the local identity, as well as to make them appealing to international markets. In this game, the translation and the digital environment play a significant role.

Regarding the overall technical aspects, there are three main challenges regarding translation methods nowadays, as specified by Vandenberg (2017): the companies are translating in silos, through different departments (which is highly inefficient and increases the localization-related problems), the lack of a good integration between different parts of the content management systems (the translated content being usually sent by email or FTP-File Transfer Protocol, downloaded, translated and emailed back and the control of the information being thus reduced) and that there is no standardized processes for translation.

Harcz (2016) argues that there are some important factors to deal with when a text is being translated from one language to another: the purpose; the target audience; the subject; the client; the given instructions; and the personal habits and preferences of the translator.

Localization process itself is a challenge that translators have to go through (de La Cova, 2016). As proposed by 
Odacioğlu (2017), the field of localization must be treated in an integrated way with translation studies. Odacioğlu (2017) proposed an integrated localization theory, integrating the linguistic, technical and technological cycles with each other.

\subsection{Motivation for Integrating Translation and Localization}

Integrating translation and localization might require the formalization of a solid theoretical background. This can be hardly achieved, due to several factors like the confidential agreements signed by the translators, which can be a methodological constrain (de la Cova, 2016). Rojo (2013) asserts that researchers have difficulties in observing and reflecting upon the type of challenges localizers have to face when carrying out different projects, and also how they cope up with these challenges.

One proposed way to increase the integration is by holding more conferences and events which discuss translation and localization (Odacioğlu, 2017). Pym (2014) maintains that, through bilateral meetings, localization can become the most dynamic paradigm of translation studies.

Technological advancements nowadays make the process of integration even more challenging. There are several technological barriers, one of them being the TM system alteration of the text through segmentation which could severely affect translators and could cause stylistic and linguistic errors; moreover, they can affect the text cohesion (de la Cova, 2016). According to Jiménez-Crespo (2010), localization is characterized by the relationship between technology and translation, therefore segmentation has to be considered one of the technological issues that localizers may face.

Localization is usually the final stage. After performing localization, the content gets published and there are times when localizers cannot do the necessary changes to adapt the content to the international markets (Herrmann \& Sachse, 2005). Also, proper feedback is difficult to receive, as there are communication barriers between localizers and authors, which are significant for content making (Fenstermacher, 2006).

One interesting approach regarding the convergence of localization and translation is the one of Mazur (2007). She combined two approaches into one: the one that perceives translation as a component of localization; and the one that perceives localization as a component of translation. She considered localization with respect to globalization, naming it "glocalization". By this framework, Mazur (2007) reconciles the two classical approaches.

When seeing translation as an aspect of localization, two theories should be referenced (Mazur, 2007): localization as text distribution suggested by Pym (2014); and the modification of Package and Content proposed by O'Hagan and Ashworth (2002). When seeing localization as part of translation, one can consider localization just as a fancy name, meaning just the process of adapting a text to the target market.

The scheme suggested by Mazur (2007) takes into account both approaches, with the observation that localization must always be considered in the large context of globalization. As explained by Robertson and White (2003), the concepts of local and global must be considered simultaneously and based on this the concept of glocalization, which was derived from "global" and "local." Robertson and White (2003) declared that what is often named local resistance against globalization is just a form of glocalization. For example, even if the final product looks local, it will still have global elements which differentiates it from other local products, thus the product has been "glocalized."

\subsection{Translation Mistakes in Localization}

Many researchers have studied translation errors in localization. For example, Diéguez-Morales and Lazo-Rodríguez (2011) studied the successes and errors found in the case of localized websites and they categorized the translation techniques according to the classification of Hurtado-Albir (2001). After some time, Jimenez-Crespo (2010) invented the holistic error typology that was dependent on the monolingual comparable mass of Spanish original and localized commercial websites. Afterwards, Reguera and Delgado (2015) focused their research on the quality of website localization and they analyzed the quality of the "Products" section from the Small and Medium-sized Enterprises (SME) corporate websites.

The most translated website is the official website of Jehovah's Witnesses "JW.org" (Hill, 2020; Tirosh, 2020). The number of the versions of the site is hard to calculate, but, as of April 2020, it is available in 1020 languages (Hill, 2020). Other websites are Google, Netflix, and Wikipedia. Google is translated in 149 languages and Netflix in 100 (base on the estimations for the end of 2019). Wikipedia, on the other hand, has a remarkable 301 language editions (Tirosh, 2020). 


\subsection{The Importance of Technology for the Integration of Translation and Localization}

In order to build bridges between translation and localization studies to address the current challenges, focusing on the informatics and communication technologies might come in handy. In the future, it is estimated that the translator's own aptitudes will be supplemented by technical tools (Alonso \& Calvo, 2015).

Also, in the last decades, a significant shift has been seen, from a culture based on text towards a culture dominated by multimedia texts and audio-visual, combining complex systems of communication (Rodrigues, 2016). This increased the difficulty of translation. According to Fuentes-Luque and Kelly (2000), the translator has started to play a significant role in marketing transactions, becoming a transmitter, a communicator of advertising messages, a cultural and linguistic mediator (Rodrigues, 2016), as well as a specialist in utilizing the latest digital tools for translation. Translation became an inter-disciplinary field, in which the role of digital technologies is tremendous.

With technology changing the ways and means in which people communicate and work, the question of how translation technology is altering the translator's work systems is very important in contemporary translation studies. The effects of the application of Computer-Assisted Translation (CAT) tools appear far-reaching and thus valid for translators and translation teachers (Pietrzak \& Kornacki, 2021). Translators can enjoy various advantages in their daily work if they are accustomed to the characteristics of CAT technology. With the advent of technology, it is possible for CAT tools to support translators in two major areas, namely proper translation and workflow management (Pietrzak \& Kornacki, 2021).

Also, according to Alonso and Calvo (2015), translators would likely get help through digitalization, which can enhance their capabilities by creating an environment using creative and learning dimensions with better outcomes; thus, it will avoid the current trends in the technologization of translation methods (Biau-Gil \& Pym, 2006).

As specified by Whalen (2014), localization consists of both translation and its adaptation to the other markets, speaking a different language. In particular, localization is also tightly connected with usability (Riippa, 2016). One example of a business depending very much on IT technology is the travel trade, which has many computerized reservation systems utilized daily by employees. As Riipa (2016) described, the deficiencies in the languages in the different functions implemented in these systems can severely affect the sales. To satisfy the customers, it is very important to take into account the usability of the online booking systems.

\subsection{User-Centred Methodologies}

It is important to culturally adapt specific products or services to certain markets in order to be successful (DePalma, 2006; Schäler, 2007) and that is one of the reasons why localization became an essential industry nowadays (de la Cova, 2016). According to de la Cova (2016), companies should start to consider localization as a strategic business decision, having a great impact on their branding and revenue.

New methodologies based on integrating translation theories with localization studies must be developed. Current translation theories are not enough for keeping up with the recent digital revolution. A more target-oriented approach is needed. In accordance with the Skopos Theory and Mänttari's Theory of Translatorial Action, equivalence should not be the main goal for the translator; rather it is to form a translated text according to the target audience, through his translation decisions (Odacioğlu, 2017). Unfortunately, these days, the localization industry forces translators to gain correspondence, because during the segment of localization, translators who are at advantage due to translation technology tools should be able to translate phases without knowing about the whole text (Odacioğlu, 2017).

Suojanen et al. (2012) benefited translation studies by introducing a new theory called User-Centred Translation (UCT). In usability engineering, this theory is parallel with User-Centred Design (UCD) (Suojanen et al., 2012). Nielsen (1993) maintained that usability has several quality components, the main being efficiency, learnability, error, memorability, and satisfaction. The purpose of user-centred translation theory is to hold up to the front the potential client throughout each stage of the translation process (Suojanen et al., 2012).

Two methodologies based on the user-centred approach are personas and heuristic evaluation. A case study that was conducted by Suokas (2020) in which the researcher chose the two simple methods (personas and heuristic evaluation) that appear to be relatively simple to apply to most translation projects. The study included research material gathered between 2015 and 2016 from BA and MA level students from two universities where user-centred translation (UCT) was applied.

The findings of the study concluded that the personas method seemed to be well-received among the students more than the heuristic evaluation method. The personas method was a simple and fun way of thinking about the 
target audience. It gave them a more concrete grasp of the readers. However, few students had problems considering the personas method to be too bland, uninspiring and sometimes too colourful, which could be either a benefit or a hindrance for translation (Suokas, 2020). The findings also concluded that the heuristic evaluation method was clearly more difficult than the personas method. But a clear benefit of heuristic evaluation was that it made translation evaluation more systematic and thorough. Some students enjoyed the specific points of focus that heuristics provided, while others found them irritating and limiting. The most evident problems with the heuristics were that the specific heuristics used in the evaluation needed further refinement. Based on the findings, the researcher recommended that UCT methods have good potential for translator training, but the specific methods require refinement (Suokas, 2020).

According to some researchers, for example Riipa (2016), the heuristic evaluation is the most efficient methods to track the usability problems for enhancing the user experience. The method was created by Nielsen and Molich (1990). Based on this method, different translation experts use pre-defined quality criteria, to evaluate the translation (Nielsen, 1994b).

\subsection{Project Management Techniques}

The user (the reader of the translation) must be central in case of each decision during a localization translation project. Also, according to Odacioğlu (2017), the translator can take various roles like post-editing, project management, marketing consultancy, language engineering, localization engineering, etc.

Esselink (2000) considered the project management as the first stage of a localization project. Also, according to Dunne and Dunne (2011), project management is considered as the basis of the language industry, but it is being constantly ignored, and reduced to an entity of academic importance and critical reflection, in the zone of translation studies. In addition, project management concentrated on translation and localization is considered a method to build bonds between the current translation scholarship and the current professional practice, offering a sound base for future analyses. Extensive information is available in the industries that have previously used the techniques of project management (for example, software engineering and construction). However, there is no research at present that studies translation and localization together in the context of project management.

Dunne (2006) observed and managed the value of translation and localization tasks. The best practice methods he found involve customer satisfaction as an aspect of quality, being unable to be defined. Therefore, the quality should be adapted to the customer demands. Dunne (2006) analyzed the traditional project management approaches, but he argued in the end that agile methodologies offer the best solutions and suggested a model for the statistical measurement of customer-related translation quality (Dunne \& Dunne, 2011).

\section{Methodology}

The present research was conducted to propose a methodology to overcome localization translation challenges in various industries.

\subsection{Research Questions}

Based on the above points of the literature review, the research questions which this paper tries to answer are the following: (a) What would be the best theoretical framework for identifying current translation problems and addressing the convergence of translation and localization according to the new developments in informatics and communication technologies? (b) How is user-centred translation capable of offering a method to avoid translation errors in the localization process? and (c) How can agile (adaptive) methodology be applied to localization projects to mitigate the localization translation problems?

\subsection{An Adaptive Method}

To answer the research questions, it was important to carry out a comprehensive review of the existing studies on translation and localization. Translation problems were seen only from a linguistic point of view until 1980, but, after that, translators have broaden the view of localization translation problems such as to include the textual, pragmatic, cultural and bilingual aspects. Hurtado-Albir (2011) validated the Process in the Acquisition of Translation Competence and Evaluation (PACTE) translation competence model and found out that the translation problems identified by the translators varied depending upon the subject's competence. The characteristics of translation competence among the subjects should be identified in solving the translation problems, by applying the right strategies and relevant knowledge (Beeby et al., 2008).

Based on the literature review, the need for a more systematic approach of translation theories and localization process will be emphasized and the types of translation problems will be derived, referencing the PACTE Group's rich point model (Jääskeläinen \& Lacruz, 2018). Using PACTE model, the pre-established prototypical 
translation problems can be identified. According to Jääskeläinen and Lacruz (2018), the translation competency model is being used to test the problem indicators by using empirical research framework, through PACTE. This model helps translator trainers and the research community by providing valuable data.

However, in order to provide a more holistic view on translation problems, the following indicators are also recommended to be addressed: procedural indicators (documented in different types of process protocols); performance indicators (errors and their feedback in various translation products); and perception indicators (predicting problematic areas beforehand).

Having determined the types of translation problems, a heuristic evaluation will be performed, by referencing different cases of translation in different major industries and using already defined quality criteria to judge the translation process. The methodology follows the one described by O'Hagan and Mangiron (2013), but with different quality criteria and the methodology will focus on the aspects of agile project management in translation and will be applied not only on the localization problems of game industry, but on different industries overall, being a descriptive and generalized approach, rather than an empirical one.

By the proposed methodology, more links between academia and industry will be established. Such attempts to establish links between academia and industry have been done in the past, in different ways. For example, the Industry-University Cooperative Research Centres Program (IUCRC) did an extensive research to build a collaboration between translation organizations and academia. Agile translation process (ATP) was used for complex innovations at the centre. The ATP fulfilled the constraints developed in the academics. ATP meets the needs of technology consumers in industry seeking a gain through the funding of the centre. By using an agile translation process and other techniques, the academic and other sectors can effectively apply such research to bring innovation to its translation industry partners (Ramanathan et. al, 2013).

Agile project management has been proven to be related to creativity at universities and other educational institutes. The communication process between the moderator and the end-user is the key to a successful application of agile techniques. The adoption of agile methodologies by higher education can lead to knowledge sharing and trust-based working among translation industries and academia.

It was proven by several researchers that there is a demand for a more solid theoretical framework in regard to the localization process (O'Hagan \& Mangiron, 2013). Nowadays, localization problems cannot be addressed without addressing the impact of technological developments on translation and without recognizing the environmental constraints of the translation itself and the phenomenon of culture clashes. According to Esselink (2000), localization is where language meets technology.

It is important to note that equivalence is not important for the translator, rather producing the translated text relevant to the approached audience, by making right decisions, is important (Odacioğlu, 2017). The user-centred translation is thus justified.

As translation work takes place usually in the form of projects and the user-centred translation is one focus of the research, an agile methodology in approaching translation projects is recommended. Also, it must be noted that translation is done nowadays through outsourcing and the capability of managing remote teams became a necessity (Dunne \& Dunne, 2011).

Building on the existing previous research, most importantly on Jääskeläinen and Lacruz (2018), O'Hagan and Mangiron (2013), Esselink (2000), Odacioğlu (2017) and Dunne and Dunne (2011), the proposed methodology can be summarized as follows:

a) Perform a literature review in order to reach a more systematic approach of translation theories and localization process, and identify the types of translation problems;

b) Revise the previous models based on the pre-defined conceptual translation errors (the so-called "rich points"), which provides an efficient way to identify the occurring translation problems;

c) Find "quality criteria" in order to evaluate localization translation projects;

d) Address localization, the influence of technological developments on translation, the environmental constraints of the translation itself and the phenomenon of culture clashes;

e) Identify the principle guidelines for a user-centred translation;

f) Justify the importance of project management techniques for any localization translation project;

g) Offer a more general model, based on the model offered by Dunne and Dunne (2011), which can incorporate a more agile approach and cover more industries, through which more links between 
academia and industry may be established and the translation theories and localization process could converge.

\section{Discussion}

\subsection{Localization Translation Challenges}

There are several ways to identify the most common localization translation challenges. For this research, the methodology was based on the PACTE's model. As discussed in the previous section, the model is built upon the thread of interdependent problem indicators: the pre-translation mark-up of anticipated problems, concrete problem indicators evidenced in screen recordings, and errors in corresponding translations. This classification is based on product and process data (Jääskeläinen \& Lacruz, 2018).

Knowing what are the most recurring problems in localization translation, the errors can be anticipated and dealt with beforehand. Jääskeläinen and Lacruz (2018) point out that this model should be supplemented with additional indicators (procedural indicators, performance indicators or perception indicators) to provide a more holistic view. According to their research, the common challenges that localization translation faces are the errors in different sub categories like bilingual extralinguistic instrumental and strategic know-how of translation.

The gaps can be identified by using triangulated data, which can be obtained through direct observation of behaviors, screen recording protocols, surveys for detecting the problems faced while translating, and through interviews. Rich points are grounded in the actual problems (screen recordings) and perceived problems (questionnaires and surveys) (Jääskeläinen \& Lacruz, 2018). Also, it is essential to notice the importance of self-feedback for a professional translator and this model is suited for self-assessing the most common localization translation problems.

The model can be applied to different industries, yielding different results. However, some common difficulties for localization translation have been encountered in all industries (Jääskeläinen \& Lacruz, 2018): the errors associated with textual levels (terminal points, pausing points in long sentences, terminology, word choice, collocation, word order, fixed expressions, tense, phrasal construction, register, issues related to the defined audience and purpose, genre conventions), or with locus (establishing equivalence, faults involving formulation in the target language, problems that involve understanding, faults involving mapping across languages and cultures, field and content-related problems).

Localization and translation challenges are often added to two different aspects, namely globalization and internationalization. Four terms are often used under the name of GILT, being an abbreviation of Globalization, Internationalization, Localization and Translation (Riipa, 2016). Therefore, according to Sandrini (2008), the globalization and internalization aspects should also be considered, when handling localization translation challenges because, after the engineering phase, the content should be easily adapted to the target market. Mazur (2007) declared that localization and GILT industry are parallel concepts. In the context of the GILT industry, translation is considered part of localization. Munday (2012) viewed localization as the process of adaptation of a service or a product involving, for example, changing some cultural symbols or some space limitations on page or screen. Similarly, in Mazur's (2007) view, translation seems to be part of localization. As Riipa (2016) stated, these definitions prove that localization includes a large range of processes.

Some of the most recent challenges in localization translation processes are the technological advancements in the last decades, which lead to a convergence of translated text with the translated audio and visual domains. For example, there are plenty of online available multiplayer games, in which the users, speaking different languages, from all over the world, interact with each other by efficiently overcoming the language barriers between them (O'Hagan \& Mangiron, 2013). The future of localization translation could highly be affected by these developments.

The translators' job has become increasingly more difficult and, when dealing with the new technological advancements, localization problems, globalization, and internationalization, they need to address the different needs of different stakeholders (their direct managers, clients, customers and so on).

\subsection{Impact of Technological Developments on Translation and Localization}

Localization is seen as one of the fastest growing sectors of translation in our world today being characterized by the dominant leadership of the Information Technology (IT) field (Jiménez-Crespo, 2013). There are many types of products which can be localized (magazines, cars, and, most importantly, the IT technology itself) (Mazur, 2007). According to Jiménez-Crespo (2013), localization as an industry originated from the end of 1970 s, from the time when US computer companies were trying to reach international markets. They started to customize 
their products (hardware and software), to adapt them to the new local markets (Riippa, 2016).

According to Pym (2014), a translator must have a good experience in cultures and languages coupled with a good knowledge of technology in order to carry out localization projects. Shortly, along with doing the act of translation, the translator can also show their abilities in localization projects, such as project management, language engineering, technical writing, localization engineering, and editing or post-editing (Pym, 2012).

Computer-Assisted Translation (CAT) technology provides an increase in speed and efficiency of translation which helps translators work better and faster. Furthermore, new technological solutions have enabled new ways and means of management, communication, and collaboration in the field of translation industry. The innovations and the continuous development of CAT technology have a huge economic impact on the services that support cross-language communication (Pietrzak \& Kornacki, 2021). However, there are certain limitations linked to CAT technology and these tools must be used as support tools, but not be used blindly.

\subsection{Principle Guidelines for a User-Centred Localization Translation Process}

Localization is tightly connected with usability. The IT industry is using the notion of usability since a significant time ago and now this notion penetrated as well into the field of translation (Riipa, 2016). Nielsen (2012) has described usability as the characteristic defining the easiness of utilizing the user interfaces.

Usability is a dish made up of five ingredients. These ingredients or quality components are learnability, memorability, efficiency, satisfaction and error (Nielsen, 1993). All of these characteristics contribute to the access of a user interface or a website, or if necessary, translation (Riippa, 2016).

Riipa (2016) points out that the system should be easy to learn (learnability); the user should be able to make the best of it after he has learned the system (efficiency); a frequent user should remember the usage of the system, even after a gap of time (memorability); the user should not make too many errors (the attribute of errors) while using the system; and they should be pleased with how the system works (satisfaction). Having a lack in one of these attributes, it is highly likely that the users will orient themselves towards another platform which is easier to use (Riippa, 2016).

According to Suojanen et al. (2012), there should be an iterative function of collecting data on end-users. Figure 1 shows the frequentative nature of user-oriented translation. First, translation that paves the way to a commission is needed, then, based on mental models (like the personas method), the translator creates a description of the target group. To create more accurate personas, the translator can use previous commissions and results of previous reception studies. After that, a translation strategy can be formed and the translation itself can begin. The expert evaluation (heuristic evaluation) can be conducted during the translation and also at the end of it. According to Suojanen et al. (2015), the phase of summing-up the entire project or the so-called "post mortem", along with the reception research, starts after the end of translation process, and also, these phases provide important information that is useful for the next steps as well as for translation itself.

Suojanen et al. (2012) state that UCT helps in providing translators with several types of techniques and tools to keep the users' needs in account. Some of these tools are the user testing (eye tracking, think-aloud protocol, interviews, questionnaires, ethnography, focus groups), mental models (intertextual reader position, personas, audience design), as well as heuristic evaluation, which is a new arrival from the usability engineering (Suojanen et al., 2012). 


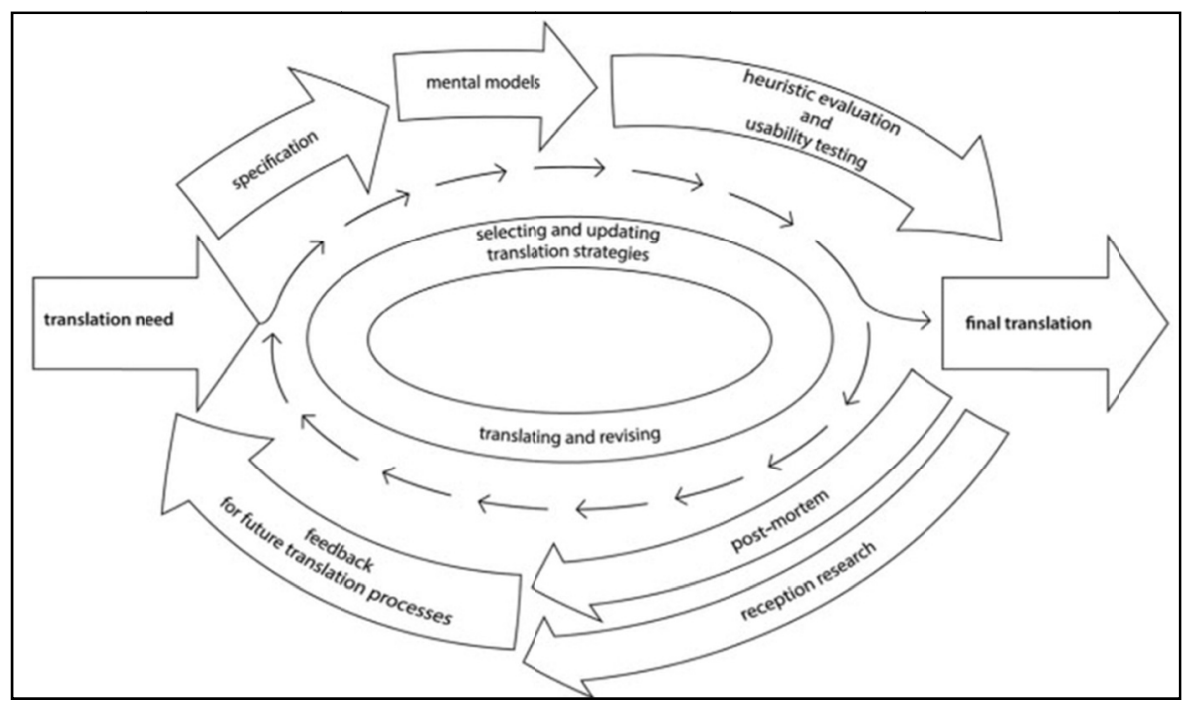

Figure 1. User-centred translation process (Suojanen et al., 2015)

\subsection{Quality Criteria to Evaluate Localization Translation Projects}

Heuristic evaluation was introduced by Nielsen and Molich (1990). The translators use heuristics or pre-defined criteria to judge the user interface (Nielsen, 1994b). The purpose of heuristic evaluation is to mobilize usability problems, to be able to correct them and enhance the overall user experience (Riippa, 2016).

A list of 10 heuristics was created by Nielsen and Molich (1990), known as Neilsen's list, in order to evaluate the user interfaces (Nielsen, 1993). Then, Purho (2000) made some changes to Nielsen's list to make it suitable for technical documentations. Otava (2013) used these lists to build a new list of heuristics for studying application of translations, with the mention that some parts of this new list did not address the usability of translation. Later, Suojanen et al. (2015) created another heuristics list for the purposes of UCT. Riipa (2016) developed a new list of heuristics for the localization translation process regarding the touristic websites. The list covers four key aspects: (1) a worldwide aspect, (2) a localization aspect, (3) a UCT aspect, and (4) a generalized usability aspect. This list was derived from Yunker's (2014) list of Web Globalization Best Practices like: Esselink (2000), O'Hagan and Mangiron's (2013), Suojanen et al. (2015), and Nielsen (1994a).

Based on previous research, mostly of Riippa (2016), the following list of heuristics for a localization translation project is proposed by the current research:

1) A comparison between the translation and specification (Why is there a need for translation and will it fulfil the requirements on the list?)

2) Match between translation and genre (Does the translation match the agreements of the genre in question? Are the graphic, audio and other multimodal components suitable for the new context?)

3) Consistency of the translation process (Is the translation consistent in style, terminology, phrase formation and registering?)

4) Legibility and readability (Do the visual elements of the translation resemble the reader's physiological capabilities and appropriate cultural guidelines? Are the users informed about the functionality of the translation by using suitable flagging for the genre in the listed queries? Are the user's efforts of interpretation, sufficiently minimized?)

5) Cognitive load and efficiency (Is the translation well-crafted enough to be easy to memorize and learned, that is, clear and comprehensible? Do the users need guidance for using the translation and if so, in which format?)

6) Satisfaction (Does the translation produce a pleasurable and/or rewarding user experience?)

7) Match between source and target texts (Has all relevant source material been translated? Is there unwanted linguistic or structural interference?)

8) Error prevention (Have potential risks of misunderstanding been minimized?)

9) Cultural adaptation of country-specific features (Have the country-specific features been adapted in a correct way? Have all country conventions or formats of the target culture been followed?) 
10) Cultural context and preferences (Are the cultural preferences of users considered important?)

According to the specifics of this research, more heuristics can be added. Also, the heuristics can be weighted differently, depending on the project. If such a heuristic evaluation is conducted early, the expected problems and errors can be easily fixed in the beginning of the localization process.

\subsection{The Importance of Project Management Techniques for Localization Translation Projects}

According to Esselink (2000), the first step to be conducted when doing a localization project is the phase of project management. A dedicated project management is necessary due to the remote locations of translation team members, and also due to the increasing number of complexities of the processes of translation projects. Clients look forward to translation agencies for their projects, and the agencies pass on their work to freelancers or specialized providers (Dunne \& Dunne, 2011).

Due to the uniqueness and unclarity of any on-going project, it is not possible to draw all of the decisions via flowcharts, checklists, decision trees and so on (Dunne \& Dunne, 2011). The linear approach is indicated when both the requirements and what must be done to fulfil them are clearly understood and specified. The incremental approach is indicated when the requirements are fully defined and specified but what must be done to fulfil them is not clear. Finally, the iterative approach is indicated when neither the requirements nor what must be done to fulfil them is clearly understood and specified (Dunne \& Dunne, 2011).

Dunne (2006) argues that agile methodologies are the best solutions for localization translation tasks, and he proposes a model for statistical management of customer-focused translation quality. Though, the acceptance of incremental and frequented approaches proved to be an exception, rather than the standard in translation and localization project management (Dunne \& Dunne, 2011).

To avoid the expected changes that happen during the localization project, an agile methodology should be followed. When the planning phase starts, the project manager collects information from client-side stakeholders (the product manager and/or project manager) about the business objectives and communicative purposes of the translation; the time, place and medium by which the target materials will be made available; high-level characteristics of the target audience; target-culture authorized or legal requirements if known; the degree of trustworthiness that the translation should show to the source text; and the intentions of the author. Collectively, these components comprise the preliminary requirements (see Figure 2, Dunne \& Dunne, 2011).

Next, the project manager engages in quality design. First, they consult with the project team to specify the contextual requirements in greater detail, including but not limited to more granular characteristics of the target audience; studied culture legal or constitutional requirements not recognized by client; cultural, linguistic and social conventions linked with the medium; as well as restrictions or boundaries imposed by the standards. The project manager then oversees a collaborative consultation between the project team and the client reviewer, in-country representatives and/or end users to specify the text-related requirements, including linguistic style, terminology, and visual style. The description of contextual and text-associated requirements must be performed side by side for each language (and perhaps for different locales within an assigned language as well, depending upon the specific task). Quality Assurance (QA) and Quality Control (QC) methods can be defined once the preliminary project quality model is complete. When the project execution phase starts, tasks should be completed and subjected to review in batches (Dunne \& Dunne, 2011).

This approach offers multiple advantages. Initially, it generates incremental error correction, which not only guarantees punctual remediation of faults, but also provides data about types and occurrence of errors. To avoid the spread of such errors in the future, this information can be provided to the project team. Like this, frequented feedback loops help the project team gradually improve its performance. In addition, feedback is provided by the incremental client review to the planning process in general, and also, to quality conditions analysis and to quality design specifically. According to Dunne and Dunne (2011), the agile methodology helps the project manager remain updated about text-related specifications and QA and QC methods highlight the emergence of new requirements, thus constantly updating the project requirements. 


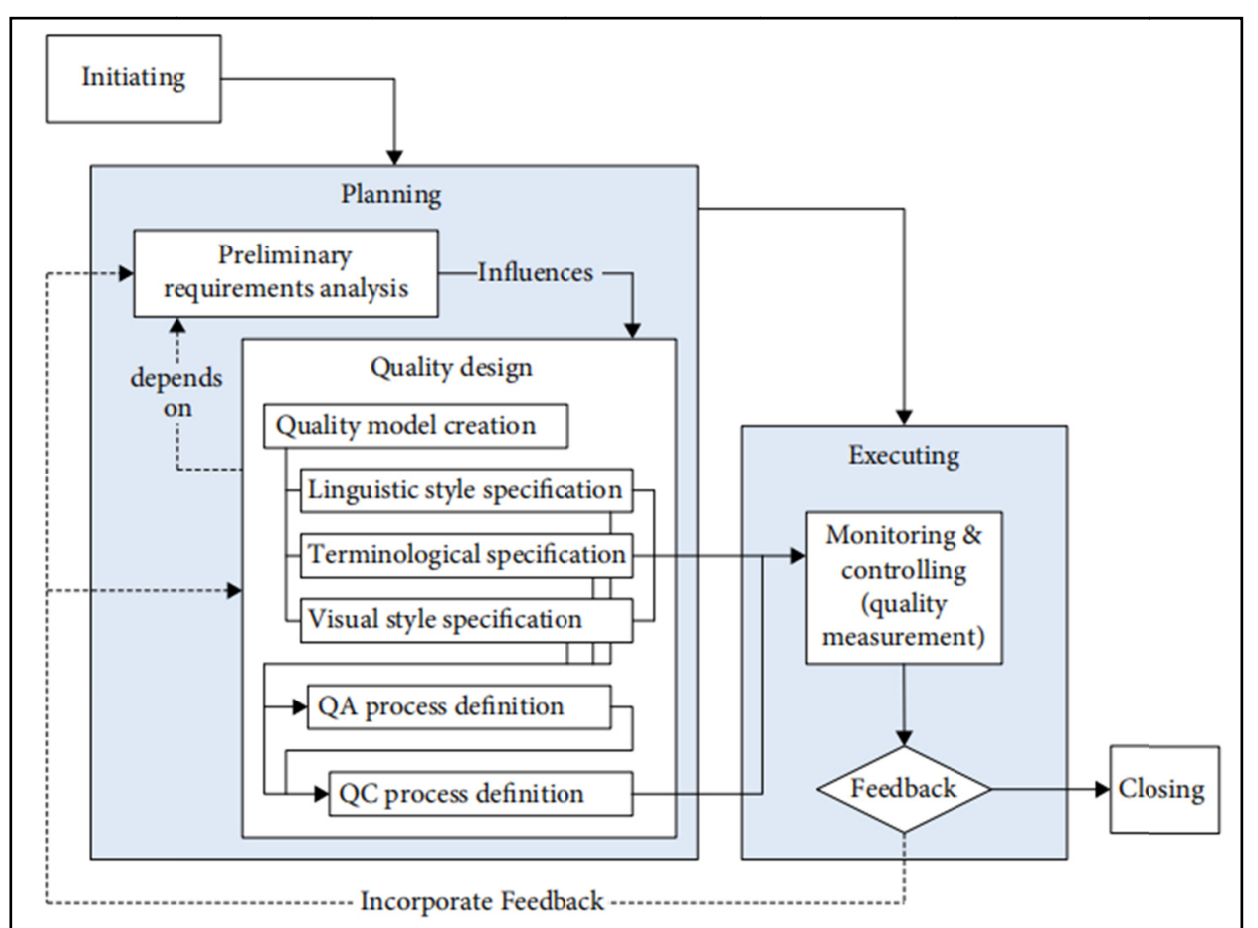

Figure 2. An agile approach to translation and localization quality management (Dunne \& Dunne, 2011)

\subsection{Converging Translation Theories and Localization Processes}

As can be noticed, the integration of translation practice with the technology is blooming (through computer programming, computer engineering, graphic design tools, translation management systems, project management systems, etc.), translators assuming different roles (e.g., project management, post-editing, marketing consultancy, localization engineering, language engineering, etc.) in the localization process, and use their 'expertise in translation practice' in integration with different fields (Odacioğlu, 2017). Therefore, translation experts can also be called localization experts. This generates the hint that the duty of translators in the localization team is not only translation. In a nut shell, the translation concept changes according to the respective field which is considered.

Odacıoglu (2017) emphasizes that localization should not be considered merely as an industrial discourse and there is a strong need to overcome the disconnection between translation studies and localization.

\subsection{The Proposed General Model}

The present research proposes a general model in approaching localization translation projects and it can be summarized as follows:

- Identify the most common localization translation challenges using the PACTE Group's rich points model, providing an efficient way to identify the occurring localization translation problems, by the means of triangulated data (obtained through direct observation, screen recording protocols, surveys for detecting the problems faced and through interviews); also, in addition to the rich points model, some procedural, performance or perception indicators can be used, according to the context;

- Identify the different roles of the translator as a technical communicator in a specific project (e.g., project management, post-editing, marketing consultancy, localization engineering, language engineering, etc.);

- Ensure the user is at the centre of each localization translation project, by assessing the following attributes: learnability, efficiency, memorability, error and satisfaction (the system for which the localization translation project is done should be easy to learn, the user should be able to make the best of it after he has learned the system, a frequent user should remember the usage of the system, even after a gap of time, the user should not make too many errors while using the system and he should be pleased with how the system works).

- Use the proposed 10 heuristics enumerated in section 4.4 in order to evaluate localization translation projects (comparison between the translation and specification; match between translation and genre; consistency of the translation process; legibility and readability; cognitive load and efficiency; satisfaction; match between source 
and target texts; error prevention; cultural adaptation of country-specific features; cultural context and preferences);

- Use the agile project management technique for most localization translation projects;

- Document the specific methodology for each localization translation project in order to create more links between academia and industry, contributing thus to the convergence of the translation theories with localization processes.

A schematic of the proposed general model is given in Figure 3, emphasizing as well on the linkage between the theory (academia) and practice (industrial applications of the proposed model).

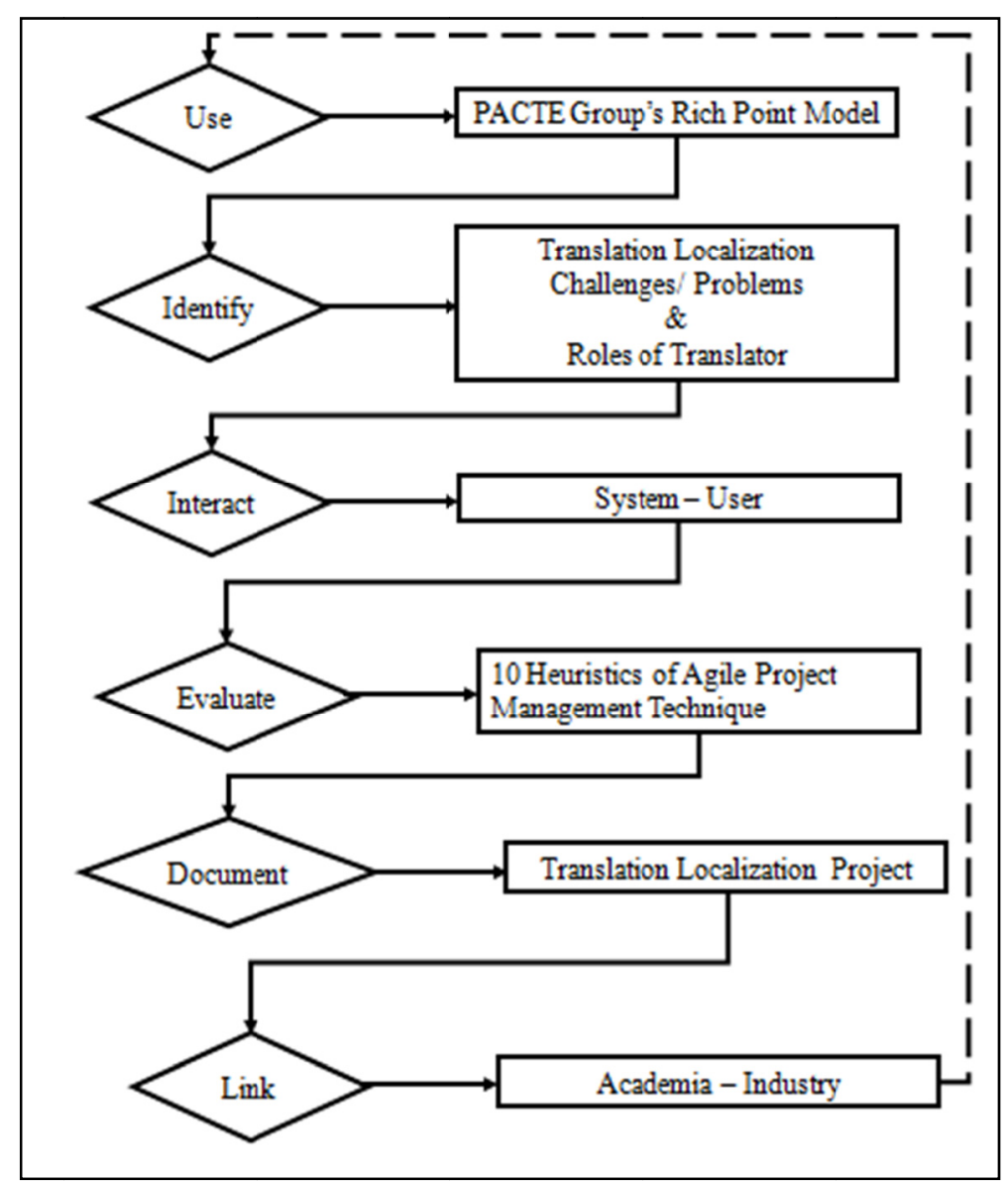

Figure 3. A proposed general model approaching localization translation projects

\section{Conclusion}

The proposed general model does not hold rigidly to a single paradigm, rather it adapts an eclectic approach. That is, it draws conclusions from several theories and ideas to gain complementary insights into the localization problems and their solutions. Though the proposed model is based on perception and reasoning, that still needs verification for its application to establish links between industry and academia.

The aim of this study was to create an adaptive methodology which can be useful for different industries in overcoming the social and technological challenges of the 21 st century, concerning translation and localization, aiming to answer the research question of what is the best methodology to adopt for relating translation theory to technology (involving the localization process) and thus to translation marketing. The research made use of the new project management techniques based on an iterative approach, as agile methodologies, in order to make localization translation projects more efficient and increase the stakeholders' satisfaction.

For this scope, it was important to analyze the past and current definitions of translation and localization, in order to build the theoretical framework for the proposed methodology. Based on the revised new definitions, 
adapted to the new socio-technological context of the present digital era, the challenges could be identified and addressed through the formulation of a new methodology.

The proposed methodology relies heavily on the previous studies conducted by Jääskeläinen and Lacruz (2018), O'Hagan and Mangiron (2013), Esselink (2000), Odacioğlu (2017), and Dunne and Dunne (2011). Several steps were considered, starting with a thorough literature review on the translation and localization concepts, on the challenges and on the importance of technological developments and the role of the digital age in the localization translation process. Then, the previous models on capturing the localization translation errors were analyzed and the model of PACTE "rich points" (Jääskeläinen \& Lacruz, 2018) was selected as being one of the best in identifying the occurring localization translation problems. Next, after mitigating the occurrence of the localization translation problems, there is a need for creating quality criteria set in order to evaluate localization translation projects. For this, a set of 10 quality criteria was chosen from the work of other researchers, especially Riipa (2016). The research has also focused on the importance of adopting a user-centred localization translation approach and on adopting an agile project management method for translation projects to assure the satisfaction of stakeholders and the necessary flexibility in adapting to any change that may occur during a localization translation project.

The framework, conceptualized in this research, would be useful in improving localization translation projects. However, further work is recommended. The framework must be validated in the future by applying it to concrete cases of localization translation projects and assess its utility and performance.

\section{References}

Abdallah, K., \& Koskinen, K. (2007). Managing trust: Translating and the network economy. Meta, 52(4), 673-687. https://doi.org/10.7202/017692ar

Alonso, E. (2016). Conflict, opacity and mistrust in the digital management of professional translation projects. Translation and Interpreting, 8(1), 19-29. https://doi.org/10.12807/ti.108201.2016.a02

Alonso, E., \& Calvo, E. (2015). Developing a blueprint for a technology-mediated approach to translation studies. Meta, 60(1), 135-157. https://doi.org/10.7202/1032403ar

Beeby, A., Fox, O., Hurtado-Albir, A., Kuznik, A., Neunzig, W., Rodríguez-Inés, P., \& Romero, L. (2008). First results of a translation competence experiment: Knowledge of translation and efficacy of the translation process. In J. Kearns (Ed.), Translator and Interpreter Training: Issues, Methods and Debates (pp. 104-126). London: Continuum International Publishing Group.

Bell, R. T. (1991). Translation and Translating: Theory and Practice. London: Longman.

Berman, A. (1989). La traduction et ses discours. Meta, 34(4), 672-679. https://doi.org/10.7202/002062ar

Biau-Gil, J. R., \& Pym, A. (2006). Technology and translation (a pedagogical overview). In A. Pym, A. Perekrestenko \& B. Starink (Eds.), Translation technology and its teaching (with much mention of localization) (pp. 5-19). Tarragona: Intercultural Studies Group, University of Rovira i Virgili.

Cronin, M. (2013). No direction home? Translation and the limits to globalization. Intercultural Communication Review, 11(1), 15-27.

de-la-Cova, E. (2016). Translation challenges in the localization of web applications. Sendebar, 27(1), 235-266. https://doi.org/10.30827/sendebar.v27i0.4942

DePalma, D. A. (2006). Quantifying the return on localization investment. In K. J. Dunne (Ed.), Perspectives on Localization (pp. 15-36). Amsterdam: John Benjamins Publishing Company. https://doi.org/10.1075/ata.xiii.03dep

Diéguez-Morales, M. I., \& Lazo-Rodríguez, R. M. (2011). The Spanish language in the Internet: Successes and errors in localized websites. Onomázein: Journal of Linguistics, Philology and Translation, 24(2), 299-326.

Dunne, K. J. (2006). Putting the cart behind the horse: Rethinking localization quality management. In K. J. Dunne (Ed.), Perspectives on Localization (pp. 95-117). Amsterdam and Philadelphia: John Benjamins Publishing Company. https://doi.org/10.1075/ata.xiii.08dun

Dunne, K. J., \& Dunne, E. S. (2011). Mapping terra incognita: Project management in the discipline of translation studies. In K. J. Dunne \& E. S. Dunne (Eds.), Translation and Localization Project Management: The Art of the Possible (pp. 1-14). Amserdam and Philadelphia: John Benjamins Publishing Company. https://doi.org/10.1075/ata.xvi.01dun

Esselink, B. (2000). A Practical Guide to Localization. Amsterdam \& Philadelphia: John Benjamins Publishing 
Company. https://doi.org/10.1075/liwd.4

Fawcett, P. (1997). Translation and Language: Linguistic Theories Explained (Translation Theories Explained). Manchester: St. Jerome Publishing.

Fenstermacher, H. (2006). Authors, localizers and language barriers. Multilingual: Language, Technology, Business, 17(1), 82.

Folaron, D. A. (2006). A discipline coming of age in the digital age. In K. J. Dunne (Ed.), Perspectives on Localization (pp. 195-219). Amsterdam and Philadelphia: John Benjamins Publishing Company. https://doi.org/10.1075/ata.xiii.16fol

Fuentes-Luque, A., \& Kelly, D. (2000). The Translator as mediator in advertising Spanish products in English-speaking markets. In A. Beeby, D. Ensinger \& M. Presas (Eds.), Investigating Translation: Selected papers from the 4th International Congress on Translation, Barcelona, 1998 (pp. 235-242). Amsterdam and Philadelphia: John Benjamins Publishing Company. https://doi.org/10.1075/btl.32.27fue

Harcz, D. B. (2016). Taking liberty as a translator. Multilingual: Language, Technology, Business, 27(1), 23-25.

Herrmann, A., \& Sachse, F. (2005). Internacionalización de aplicaciones de software. In D. Reineke (Ed.), Traducción y localización: Mercado, gestión y tecnologías (pp. 45-70). Las Palmas, de Gran Canaria: Anroart.

Hill, C. (2020). What is the Most Translated Website in the World? Retrieved March 1, 2021, from The WordPoint:

https://thewordpoint.com/blog/worlds-most-translated-website\#: :text=In\%20accordance\%20with\%20this \%20definition,linguistic\%20variety\%20is\%20very\%20impressive

Hurtado-Albir, A. (2001). Traducción y Traductología: Introducción a la Traductología. Madrid: Ediciones Cátedra.

Hurtado-Albir, A. (2011). Results of the validation of the PACTE translation competence model: Translation problems and translation competence. In C. Alvstad, A. Hild \& E. Tiselius (Eds.), Methods and Strategies of Process Research: Integrative Approaches in Translation Studies (pp. 317-343). Amsterdam: John Benjamins. https://doi.org/10.1075/btl.94.22pac

Jäskeläinen, R., \& Lacruz, I. (2018). Translation - cognition - affect - and beyond: Reflections on an expanding field of research. In I. Lacruz \& R. Jääskeläinen (Eds.), Innovation and Expansion in Translation Process Research (pp. 1-16). Amsterdam and Philadelphia: John Benjamins Publishing Company. https://doi.org/10.1075/ata.18.01jaa

Jiménez-Crespo, M. A. (2010). The intersection of localization and translation: A corpus study of Spanish original and localized web forms. Translation and Interpreting Studies, 5(2), 186-207. https://doi.org/10.1075/tis.5.2.03jim

Jiménez-Crespo, M. A. (2013). Translation and Web Localization. London and New York: Routledge. https://doi.org/10.4324/9780203520208

Koller, W. (1979). Introduction to Translation Studies. Heidelberg: Quelle \& Meyer Publishing Company.

Ladmiral, J.-R. (1979). Traduire: Theorèmes pour la traduction. Paris: Payot.

Lakó, C. (2014). Localizing Websites: Shifting Focus onto the End-User. Iași, Romania: Unpublished Ph.D. thesis, Alexandru Ioan Cuza University.

Lommel, A. (2003). The Localization Industry Primer (2nd ed.). Féchy: Localization Industry Standards Association (LISA).

Mazur, I. (2007). The metalanguage of localization: Theory and practice. Target, 19(2), 337-358. https://doi.org/10.1075/target.19.2.11maz

Munday, J. (2012). Introducing Translation Studies (3rd ed.). London and New York: Routledge. https://doi.org/10.4324/9780203121252

Nielsen, J. (1993). Usability Engineering. San Francisco: Morgan Kaufmann. https://doi.org/10.1016/B978-0-08-052029-2.50007-3

Nielsen, J. (1994a). 10 Usability Heuristics for User Interface Design. Retrieved March 2021, 1, from Nielsen Norman Group: https://www.nngroup.com/articles/ten-usability-heuristics/ 
Nielsen, J. (1994b). How to Conduct a Heuristic Evaluation. Retrieved March 1, 2021, from Nielsen Norman Group: http://www.nngroup.com/articles/how-to-conduct-a-heuristic-evaluation

Nielsen, J. (2012). Usability 101: Introduction to Usability. Retrieved March 1, 2021, from Nielsen Norman Group: https://www.nngroup.com/articles/usability-101-introduction-to-usability/

Nielsen, J., \& Molich, R. (1990). Heuristic evaluation of user interfaces. In J. Carrasco \& J. Whiteside (Eds.), Seattle. WA: Association for Computing Machinery (ACM) Press. https://doi.org/10.1145/97243.97281

Nord, C. (1997). Translating as a Purposeful Activity: Functionalist Approaches Explained. Manchester: St. Jerome Publishing.

Nord, C. (2005). Text Analysis in Translation: Theory, Method, and Didactic Application of a Model for Translation-Oriented Text Analysis. Amsterdam and New York: Rodopi Publishing Company.

O'Hagan, M., \& Ashworth, D. (2002). Translation-mediated Communication in a Digital World: Facing the Challenges of Globalization and Localization. Clevedon: Multilingual Matters.https://doi.org/10.21832/9781853595820

O'Hagan, M., \& Mangiron, C. (2013). Game Localization: Translating for the Global Digital Entertainment Industry. Amsterdam and Philadelphia: John Benjamins Publishing Company. https://doi.org/10.1075/btl.106

Odacioğlu, M. C. (2017). Integrated Localization Theory of Translation Studies. International Journal of Comparative Literature \& Translation Studies, 5(4), 24-30. http://dx.doi.org/10.7575/aiac.ijclts.v.5n.4p.24

Otava, A. (2013). Focus on the Audience: Three Cases of User-centered Translation. Tampere: Unpublished M.A. Thesis, University of Tampere, Finland.

Pergnier, M. (1993). Les fondements socio-linguistiques de la traduction. Lille: Lille University Press.

Pietrzak, P., \& Kornacki, M. (2021). Using CAT Tools in Freelance Translation: Insights from a Case Study. New York: Routledge. https://doi.org/10.4324/9781003125761

Purho, V. (2000). Heuristic inspections for documentation: 10 recommended documentation heuristics. STC Usability SIG Newsletter, 6(4). Retrieved December 1, 2020, from https://stcsig.org/usability/newsletter/0004-docsheuristics.html

Pym, A. (2012). On Translator Ethics (H. Walker, Trans.). Amsterdam and Philadelphia: John Benjamins Publishing Company. https://doi.org/10.1075/btl.104

Pym, A. (2014). Exploring Translation Theories (2nd ed.). London and New York: Routledge.

Ramanathan, J., Ramnath, R., Herold, M. J., \& Wierwille, B. J. (2013). An Agile Translation Process for complex innovations: An Industry/University Cooperative Research Center case study (pp. 1-7). IEEE Frontiers in Education Conference (FIE). Oklahoma City, OK, USA: IEEE. https://doi.org/10.1109/FIE.2013.6685094

Reguera, A. M., \& Delgado, C. R. (2015). La localización de la sección "productos" en sitios web de empresas exportadoras agroalimentarias. Online Translation Journal, Special Issue: New Insights into Specialised Translation.

Richards, I. A. (1953). Toward a theory of translation. In A. F. Wright (Ed.), Studies in Chinese Thought (pp. 147-162). Chicago: University of Chicago Press.

Riippa, J. (2016). User-Centered Translation in Website Localization: Overall Usability of the Finnish Country Site of Hotels.com. Vaasa: Unpublished Master's Thesis, University of Vaasa.

Robertson, R., \& White, K. (2003). Globalization: Critical Concepts in Sociology. London: Routledge.

Rodríguez, C. V. (2016). Globalization and localization in advertising translation: A love-hate relationship? Revista de Lenguas para Fines Especificos, 22(2), 130-153. https://dx.doi.org/10.20420/rlfe.2016.320

Rojo, A. (2013). Diseños y métodos de investigación en traducción. Madrid: Síntesis.

Sandrini, P. (2008). Localization and translation. In H. Gerzymisch-Arbogast, G. Budin \& G. Hofer (Eds.), LSP Translation Scenarios: Selected Contributions to the EU Marie Curie Conference Vienna 2007 (pp. 167-192). Norderstedt: ATRC Group.

Saussure, F. D. (1972). Cours de linguistique generale. Paris: Payot (Original work published 1916).

Schäler, R. (2007). Reverse localisation. Localisation Focus: The International Journal of Localisation, 6(1), 39-48. 
Shveitser, A. D. (1987). Ubersetzung und Linguistik. Berlin: Akademie.

Sprung, R. C. (2000). Translating into Success: Cutting-edge Strategies for Going Multilingual in a Global Age. Amsterdam and Philadelphia: John Benjamins Publishing Company. https://doi.org/10.1075/ata.xi

Suojanen, T., Koskinen, K., \& Tuominen, T. (2012). Käyttäjäkeskeinen kääntäminen. Tampere: Tampere Studies in Language, Translation and Literature, B1.

Suojanen, T., Koskinen, K., \& Tuominen, T. (2015). User-Centered Translation (Translation Practices Explained). Abingdon, NY: Routledge. https://doi.org/10.4324/9781315753508

Suokas, J. (2020). Testing Usability Methods in Translation Courses: Personas and Heuristic Evaluation. Current Trends in Translation Teaching and Learning E, 7(1), 4-38. https://doi.org/10.51287/cttl_e_2020_2_juho_suokas

Tirosh, O. (2020). What is the World's Most Translated Website? Retrieved March 1, 2021, from Tomedes: Smart Human Translation: https://www.tomedes.com/translator-hub/most-translated-website.php

Vandenberg, R. (2017). Solutions for the top three localization challenges. Retrieved March 1, 2021, from Multilingual: https://multilingual.com/solutions-top-three-localization-challenges/

Vermeer, H. J. (2001). A Framework for a General Theory of Translation. Shanghai: Shanghai Foreign Language Education Press.

Whalen, J. (2014). What is Localization (L10N)? Retrieved March 1, 2021, from Lionbridge Translation: $\mathrm{http} / / /$ content.lionbridge.com/localization-110n/?utm_source=LinkedIn\&utm_medium=social\&utm_campai gn=What $\% 20$ is $\% 20$ Localization $\% 20 \% 28 \mathrm{~L} 10 \mathrm{~N} \% 29 \% 3 \mathrm{~F}$

Yunker, J. (2014). Beyond Borders: Web Globalization Strategies. Indianapolis: New Riders.

\section{Copyrights}

Copyright for this article is retained by the author, with first publication rights granted to the journal.

This is an open-access article distributed under the terms and conditions of the Creative Commons Attribution license (http://creativecommons.org/licenses/by/4.0/). 